\title{
Doença crônica infantojuvenil: vínculo profissional- família para a promoção do apoio social
}

\author{
Chronic disease in children and adolescents: professional-family bond for the promotion \\ of social support \\ Enfermedad crónica infanto-juvenil: vínculo professional-familia para la promoción \\ del apoyo social
}

Como citar este artigo: Machado NA, Nóbrega VM, Silva MEA, França DBL, Reichert APS, Collet N. Doença crônica infantojuvenil: vínculo profissional-família para a promoção do apoio social. Rev Gaúcha Enferm. 2018;39:2017-0290. doi: https://doi. org/10.1590/1983-1447.2018.20170290.
Universidade Federal da Paraiba (UFPB), Centro de Ciências da Saúde, Programa de Pós-Graduação em Enfermagem. João Pessoa, Paraiba, Brasil.

- Universidade Federal da Parába (UFPB), Centro de Ciências da Saúde, Departamento de Enfermagem em Saúde Coletiva. João Pessoa, Parába, Brasil.

\section{Amanda Narciso Machado ${ }^{a}$ Vanessa Medeiros da Nóbrega ${ }^{a}$ Maria Elizabete de Amorim Silva ${ }^{a}$ Daniele Beltrão Lucena de França ${ }^{a}$ Altamira Pereira da Silva Reicherta ${ }^{a, b}$ Neusa Collet ${ }^{\mathrm{a}, \mathrm{b}}$}

RESUMO

Objetivo: Analisar o vínculo na promoção do apoio social ofertado pela equipe multiprofissional de saúde às famílias durante a hospitalização de crianças/adolescentes com doença crônica.

Método: Pesquisa qualitativa, realizada em unidade pediátrica de um hospital público da Paraíba, de novembro de 2012 a julho de 2013, com quatorze profissionais de saúde, por meio de entrevista semiestruturada. A interpretação dos dados foi mediada pela análise temática.

Resultados: 0 vínculo equipe-família é promovido pelo diálogo e escuta, reconhecidos como fontes de apoio social. Evidenciaram-se dificuldades como a fragilidade na comunicação e interação entre equipe-família; lacunas na organização do processo de trabalho e falta de infraestrutura hospitalar, que interferem na oferta de apoio. Como caminhos para superar os obstáculos, evidenciou-se 0 seguimento após alta e fortalecimento do vínculo equipe-família.

Conclusões: 0 apoio social satisfatório influencia o enfrentamento da doença crônica infantojuvenil e, para sua oferta, o vínculo e o acolhimento são indispensáveis.

Palavras-chave: Apoio social. Doença crônica. Família. Relações profissional-família. Criança.

\section{ABSTRACT}

Objective: To analyze the bond in social support delivery by a multidisciplinary team to families during the hospital stay of children/ adolescents with chronic disease

Method: Qualitative research, conducted in the pediatric unit of a public hospital in Paraíba State, Brazil, from November 2012 to July 2013, involving fourteen health professionals, by means of a semi-structured interview. The data were subjected to thematic analysis. Results: The bond between the team and the family is promoted by dialogue and listening, which are recognized sources of social support. The encountered difficulties that interfered with the delivery of social support were weakness in team-family communication and interaction, gaps in the organization of the work process and lack of hospital infrastructure. Possible approaches to overcome these obstacles were follow-up beyond hospital discharge and a stronger team-family bond.

Conclusion: Satisfying social support has an influence on coping with chronic disease in children and adolescents, and its proper delivery relies upon bonding and embracement.

Keywords: Social support. Chronic disease. Family. Professional-family relations. Child.

\section{RESUMEN}

Objetivo: Analizar el vínculo en la promoción del apoyo social ofertado por el equipo multiprofesional de salud a las familias durante la hospitalización de niños/adolescentes con enfermedad crónica.

Método: Investigación calitativa, realizada en unidad pediátrica de un hospital público de Paraíba, de noviembre de 2012 a julio de 2013, con catorce profesionales de salud, por medio de entrevista semiestructurada. La interpretación de los datos fue mediada por el análisis temático.

Resultados: El vínculo profesional-familia es promovido por el diálogo y la escucha, reconocidos como fuentes de apoyo social. Se evidenció dificultades como la fragilidad en la comunicación e interacción entre equipo-familia; las lagunas en la organización del proceso de trabajo y la falta de infraestructura hospitalaria, que interfieren en la oferta de apoyo. Como caminos para superar los obstáculos, se evidenció el seguimiento después del alta y fortalecimiento del vínculo equipo-familia.

Conclusiones: El apoyo social satisfactorio influencia el enfrentamiento de la enfermedad crónica infanto-juvenil y, para su oferta, el vínculo y el acojimiento son indispensables.

Palabras clave: Apoyo social. Enfermedad crónica. Familia. Relaciones profesional-familia. Niño 


\section{—INTRODUÇÃO}

A doença crônica na infância e na adolescência pode ocasionar desequilíbrio familiar, tendo em vista as alterações que desencadeiam na dinâmica da família e na vida da criança/adolescente, e tem sido tema relevante para a reflexão acerca da organização do processo de trabalho em saúde nos diferentes níveis de atenção(1).

Levantamento realizado em 2010 no Brasil identificou no mínimo uma doença crônica em 9,1\% entre as crianças de 0 a 4 anos; 9,7\% entre 6 a 13 anos e $11 \%$ entre os adolescentes de 14 a 19 anos $^{(2)}$. Diante dessa realidade, é fundamental o apoio dos profissionais aos familiares dessas crianças e adolescentes para que possam enfrentar a tarefa árdua de lidar com a doença crônica. Esse apoio faz-se necessário porque a doença crônica impacta as relações familiares, o emocional, o financeiro, a saúde de toda a família ao exigir adaptações em seu cotidiano para superar as implicações que esta traz consigo ${ }^{(1)}$. Estudo realizado na Holanda também enfatiza a importância de os profissionais de saúde capacitarem os pais para o manejo da doença, a fim de que consigam equilibrar sua vida diante das responsabilidades com o cuidado do filho com doença crônica ${ }^{(3)}$.

O apoio social implica na ajuda como suporte de afeto, confiança e empatia, podendo essa oferta ser de apoio emocional, material e afetivo realizado pela rede social do indivíduo, com o propósito de que a pessoa apoiada sinta-se satisfeita com esse auxílio(4). O apoio ofertado pela equipe multiprofissional de saúde às famílias é um dos tipos de apoio social e contribui para o melhor manejo das implicações advindas do adoecimento crônico. Contudo, este suporte precisa ser reconhecido pela família como benéfico para a sua qualidade de vida, com reflexos no cuidado prestado à criança/adolescente ${ }^{(5)}$. Assim, é importante que a equipe saiba se o apoio que tem oferecido à família atende às suas necessidades e expectativas.

Nesse sentido, é imprescindível a equipe multiprofissional de saúde pautar suas ações pela escuta qualificada e estar atenta às reflexões, experiências e saberes que os envolvidos com a doença crônica trazem consigo, para priorizar suas necessidades e não se restringir apenas às imposições terapêuticas ${ }^{(6)}$, pois a escuta qualificada prescinde a formação de vínculos.

O conceito de vínculo é polissêmico e, neste estudo, será "compreendido como uma relação interpessoal, estabelecida ao longo do tempo entre usuário e profissional de saúde, caracterizada por confiança, responsabilidade e partilha de compromissos ${ }^{\prime \prime(7)}$.

Nessa perspectiva, evidencia-se a relação escuta-confiança para a formação de vínculo. Em contrapartida, estudo aponta que a falta da escuta atenta e do diálogo com quem procura os serviços de saúde pode fragilizar ou até segregar o vínculo, trazendo prejuízos na produção do cuidado ${ }^{(8)}$, mostrando desinteresse em estabelecer vínculo.

Portanto, a escuta atenta é uma ferramenta importante no cuidado à criança/adolescente com doença crônica e os profissionais precisam reconhecer a sua importância e seu papel na rede de apoio a essa população, pois, quando apoiados, a família e a criança/adolescente irão sentir-se fortalecidos para o enfrentamento das implicações desencadeadas pelo adoecimento crônico. Por outro lado, a ausência desse suporte pela equipe de saúde torna ainda mais difícil a situação vivenciada por essa população(6).

Nesse contexto, este estudo busca trazer um olhar sensível para profissionais de saúde que atuam em nível hospitalar, direcionado para àqueles que vivenciam o sofrimento do adoecimento crônico, possibilitando, assim, reflexões e mudanças no seu processo de trabalho. Entende-se que, para a construção do cuidado à criança/adolescente e sua família balizada na integralidade, no respeito à vida e à cidadania, é fundamental que os profissionais de saúde conheçam as especificidades dessa população, a fim de reorganizarem seu processo de trabalho a partir da realidade concreta desses sujeitos.

Diante do exposto, questionou-se: o vínculo profissional-família tem ofertado apoio social satisfatório à família durante a hospitalização de crianças/adolescentes com doença crônica? Portanto, objetivou-se analisar o vínculo na promoção do apoio social ofertado pela equipe multiprofissional de saúde às famílias durante a hospitalização de crianças/adolescentes com doença crônica.

\section{—MÉTODO}

Pesquisa qualitativa, exploratório-descritiva, desenvolvida em uma unidade de internação pediátrica de um hospital público da Paraíba-Brasil, no período de novembro de 2012 a julho de 2013. A escolha do hospital deve-se ao fato de o mesmo ser referência para o tratamento de doenças crônicas e raras, além de campo de formação de alunos da área da saúde. Tais aspectos mobilizam a discussão do papel da equipe multiprofissional na assistência em saúde como integrante da rede de apoio social de crianças/adolescentes com doenças crônicas e suas famílias, tendo em vista o compromisso com a melhoria da qualidade no cuidado.

Participaram da pesquisa quatorze profissionais da equipe multiprofissional da unidade pediátrica do referido hospital, cujo processo de trabalho tem como filosofia a modalidade de trabalho em equipe. Essa equipe é formada por enfermeiros, médicos, assistentes sociais, nutricionistas, 
técnicos de enfermagem e psicólogos responsáveis pelo cuidado integral de as crianças e adolescentes internados da unidade em estudo, conta, ainda, com suporte ambulatorial. A seleção dos integrantes ocorreu de maneira aleatória, atendendo aos seguintes critérios de inclusão: atuar na unidade em estudo há mais de um ano e atender crianças/adolescentes com doença crônica. Foram excluídos os profissionais que se encontravam afastados do trabalho por motivo de férias, licenças ou outros no período da coleta de dados. Acredita-se que o fato de atuar na unidade há mais de um ano amplia o olhar do profissional para identificar as necessidades singulares nas condições crônicas infantojuvenis.

A coleta dos dados empíricos ocorreu por meio da entrevista semiestruturada $a^{(9)}$ que, após anuência dos participantes, foi gravada e transcrita na íntegra, conduzida pela questão norteadora: Que ações você desenvolve no seu cotidiano, e que, na sua percepção, fortalecem o vínculo e fornecem algum tipo de apoio às famílias de crianças/adolescentes com doença crônica? Todas as entrevistas foram realizadas na unidade pediátrica do Hospital em estudo, em uma sala privativa, no horário estipulado pelo participante que era contatado anteriormente a realização da entrevista. Para a identificação das categorias profissionais do serviço, foi realizado visita antes do início da coleta de dados para levantamento dessa informação. O encerramento da coleta seguiu o critério de saturação teórica, quando o material empírico permite o delineamento compreensivo do objeto de estudo ${ }^{(10)}$.

Os dados foram interpretados por meio da análise temática. Inicialmente realizou-se uma organização do material, com leitura exaustiva e repetida dos textos, apreendendo-se os núcleos de sentido originados pela leitura transversal. Em seguida, agrupou-se os temas relevantes para proceder a análise final ${ }^{(9)}$.

A pesquisa foi aprovada pelo Comitê de Ética em Pesquisa sob CAAE $N^{\circ}$ 0466.0.000.126-11. Todos os participantes assinaram o Termo de Consentimento Livre e Esclarecido conforme determina a Resolução 466, de 12 de dezembro de 2012 do Conselho Nacional de Saúde. Nos resultados, os relatos estão identificados pela letra E entre parênteses, acompanhado pelo numeral ordinal que se refere à ordem de realização das entrevistas.

\section{— RESULTADOS E DISCUSSÃO}

Participaram da pesquisa cinco Enfermeiras, duas Técnicas de Enfermagem, duas Auxiliares de Enfermagem, uma Pediatra, uma Médica Residente de Pediatria, uma Psicóloga, uma Nutricionista e uma Assistente Social, com idades entre 26 e 61 anos. O tempo médio de atuação em pediatria foi de aproximadamente 13 anos.
A partir da interpretação do material empírico foram identificadas diversas temáticas que permitiram a construção dos seguintes núcleos de sentido: 'Vínculo como fonte de apoio social', 'Óbices na formação de vínculo que interferem na oferta de apoio social' e 'Caminhos para superar os obstáculos na formação de vínculos para oferta de apoio social satisfatório.

\section{Vínculo como fonte de apoio social}

A forma de organização do trabalho no ambiente hospitalar é uma estratégia que pode minimizar o sofrimento decorrente da hospitalização ou intensificá-lo, a depender do foco da atenção da equipe de saúde.

[...] você parar em uma enfermaria, ficar conversando um pouco, que às vezes elas (mães) precisam, mais a mãe do que a criança. É você chegar e dar bom dia, dar um sorriso. [...] Um pouquinho de si para dar, para eles se sentirem melhor (E10).

A nossa função é ouvir mesmo. [...] tem gente que não gosta de envolvimento com o paciente. Eu abraço, "precisa de um abraço?!" Às vezes um abraço cura aquela angústia, aquela tristeza. Às vezes o paciente precisa que você segure na mão dele, converse com ele e dê uma injeção de ânimo nele. [...] Remédio é atenção, é carinho (E1).

Em consonância com este achado, estudo ${ }^{(8)}$ afirma que um serviço que não promove acolhimento e não fornece o apoio social necessário pode trazer sofrimento à criança/ adolescente e sua família, além de outras dificuldades no enfrentamento da doença, como a exacerbação de sentimentos de tristeza e medo, relação conflituosa entre profissionais e a família, além do sentimento de subordinação aos serviços de saúde. Ademais, ao acolher as famílias com responsabilidade e resolutividade no hospital, é possível a formação de vínculos, minimizando as implicações geradas pela doença e contribuindo para que a família reconheça os profissionais como fonte de apoio.

Pelo tempo de permanência no hospital e pelas recorrentes hospitalizações, os profissionais e as famílias de crianças e adolescentes com doenças crônicas têm oportunidades de estabelecer uma relação de proximidade, melhorando a adaptação à situação vivenciada. Para responsabilizar-se pelo cuidado ao outro no cotidiano é preciso estar aberto, querer estabelecer vínculo com a família para que esta tenha confiança no profissional. O diálogo, pautado pela escuta atentiva, é uma ferramenta importante de trabalho da equipe multiprofissional. 
Quando conseguimos convencê-los disso, de que estamos tentando fazer o melhor, talvez não seja dentro da expectativa dele, mas é o que pode ser oferecido [...] Isso dá tranquilidade à família e a nós também (E6).

Tentamos fornecer o máximo de informação possível, porque o que você quer saber realmente o que seu filho tem. E se cada um tenta do seu jeito explicar a doença do seu filho, é muito mais fácil eles se sentirem mais bem apoia$\operatorname{dos}[\ldots](E 3)$.

A comunicação de fato acontece quando eu te escuto e você me escuta. É a mesma coisa com a mãe, com a família, ele vai se sentir mais confiante na eficácia do tratamento. Se eu ainda não tenho a resposta [...] eu tenho que dizer para a família os passos que estão sendo dados na busca do tratamento daquela criança (E11).

O apoio social fornecido pela equipe multiprofissional é parte essencial do processo de trabalho em saúde e, para que este ocorra, faz-se necessário que haja qualificação na escuta, formação de vínculos entre familiares/profissionais, maior acesso aos serviços oferecidos. Estudo ${ }^{(11)}$ evidencia que essas ferramentas permitem criar um contexto interacional de experiências em que possam ser expressas as vivências e os saberes de cada família na sua singularidade. Essas ações são pautadas na responsabilização e na resolutividade das demandas da criança/adolescente e sua família(11).

Portanto, o vínculo entre a família e os profissionais é essencial para a identificação das demandas de apoio, e a equipe deve trabalhar em sua formação desde o primeiro contato. Esse é um processo gradativo e construído por meio de uma relação mais próxima com o cuidador familiar durante a hospitalização. Ao se sentir acolhida nesse ambiente, a família passa a confiar no profissional para confidenciar seus medos, angústias e necessidades. Em contraposição, revisão integrativa da literatura(12) evidenciou que o estabelecimento de relações interpessoais de maneira técnica e formal, desencadeiam dificuldades na comunicação e nas ações de cuidado.

O profissional que no cotidiano se mostra acessível e aberto ao diálogo, passa a ser, para o familiar cuidador, sua principal fonte de apoio durante a hospitalização, pois sempre que precisar de informações, conselhos e suporte emocional para lidar com as situações circunstanciais ou sanar dúvidas é a esse profissional que a família irá recorrer. A empatia, o cuidado solidário e o apoio fornecido pelos profissionais assinalam o vínculo estabelecido com a família, fortalecendo o reconhecimento da equipe como parte da sua rede de apoio:
[...] as famílias e as crianças criam vínculos com o profissional e eles confiam em nós, acreditam em nós, sentem-se seguros quando assistidos pelo profissional com quem ele cria vínculo. Então acho que esse vínculo ele passa a ser uma fonte de apoio. [...] Esses momentos de diálogo, [...] nos aproxima, permite que possamos apoiá-los por tudo que eles estão precisando (E5).

Estudo ${ }^{(13)}$ assevera que ao promover uma escuta atenciosa, ouvindo o familiar cuidador, suas preocupações, e demonstrando uma postura cuidativa, os profissionais instituem uma relação de proximidade e confiança com a família, o que facilita a firmação do vínculo. Diante dessas atitudes, o familiar cuidador passa a sentir-se verdadeiramente seguro, expressando suas necessidades de apoio e confiando no profissional, possibilitando o alcance da assistência integral ao binômio criança/adolescente e família.

O diálogo aproxima a equipe da família fortalecendo o vínculo existente, ampliando o cuidado e o apoio fornecido pelos profissionais, pois quanto mais confiança a família tem no profissional, mais aberta estará para revelar suas reais demandas de cuidado. Entretanto, quando a relação equipe-família está focada em ações tecnoassistenciais, os vínculos são superficiais limitando as possibilidades de estabelecer um encontro genuíno de cuidado cujo apoio fornecido seja satisfatório para as famílias.

(Énecessário) firmar realmente um vínculo com a familia e com o paciente. Muitas vezes você não tem tempo de formar esse vínculo, torna-se uma relação muito superficial, muito profissional, e deixa de ter realmente o vínculo que é necessário para que esse paciente melhore (E6).

Em contrapartida, estudo(14) sobre os cuidados e as concepções da equipe de enfermagem frente à família acompanhante da criança hospitalizada evidenciou que o tempo de permanência no hospital, mesmo sendo um ambiente estranho e desagradável, favorece o vínculo com as crianças e suas famílias, ultrapassando o cuidado técnico.

Além disso, ao estabelecer vínculo com as famílias, a equipe de saúde tem elementos para prestar cuidado humanizado e integral, transformando seu processo de trabalho na perspectiva da corresponsabilização entre equipe e família, em que esta participa ativamente do projeto terapêutico(15). Nesse processo, o profissional, para ser reconhecido como fonte de apoio social, necessita conhecer as singularidades da família para contemplá-las na perspectiva do cuidado.

Orientar a alta hospitalar de acordo com a condição socioeconômica, fazer o cardápio para ser seguido em casa (E14). 
Portanto, independentemente do tempo de atuação em pediatria, os participantes do presente estudo reconhecem a importância da escuta, do diálogo e do vínculo como formas de apoio social efetivo à família de crianças/ adolescentes com doença crônica. Entretanto, nem sempre as ações da equipe multiprofissional convergem na perspectiva de estabelecer vínculos que forneçam apoio satisfatório às famílias de crianças e adolescentes com doenças crônicas durante sua permanência no hospital. Quando isso acontece, evidenciam-se óbices que obstaculizam a formação de vínculo, fragilizando o apoio fornecido pela equipe à família.

\section{Óbices na formação de vínculo que interferem na oferta de apoio social}

A organização do processo de trabalho da equipe multiprofissional depende das concepções dos profissionais de saúde sobre o significado do trabalho em equipe e da sua importância para o alcance da qualidade da assistência, com atenção integral às necessidades apresentadas pela criança/adolescente com doença crônica e pelo cuidador familiar.

No cotidiano dos serviços, os profissionais identificaram dificuldades que interferem nos vínculos com a família e, portanto, na oferta de apoio. Dentre essas, a fragilidade na interação, na interdisciplinaridade e na comunicação entre os membros da equipe; a sobrecarga de trabalho, com consequente redução do tempo disponível para estar junto com o cuidador/criança/adolescente; a burocracia dos serviços de saúde e a falta de privacidade, aspectos que fragilizam a dinâmica do serviço e a qualidade da assistência:

Aqui não tem uma equipe multiprofissional. Aqui tem uma equipe de vários profissionais [...] Equipe multiprofissional é aquela que tem vários profissionais que todo mundo fala a mesma língua. [...] Quando fosse passar na visita, passar essa equipe, porque quando acontecesse alguma coisa com a criança, todo mundo já estava sabendo (E2).

Eu acho que no geral o que falta mesmo é esse olhar diferente no hospital todo para atender mais especificamente as necessidades. A equipe que fica aqui, muitas vezes fica de mãos atadas, tenta resolver, mas não consegue por uma questão de tempo mesmo, de burocracia dentro do hospital (E4).

Há pouca comunicação entre a equipe. E se não há essa comunicação entre a equipe, não tem como ter um apoio multiprofissional. São apoios isolados. São ações isoladas.
[...] O ideal seria que todo mundo falasse a mesma linguagem, para não criar confusão na cabeça das mães (E5).

A ausência do trabalho em equipe fragiliza a assistência ao binômio e o processo de trabalho. Quando não há comunicação entre os membros da equipe, o vínculo com a família fica fragilizado comprometendo o apoio social ofertado. Esses achados estão em consonância com estudo que afirma que as fragilidades na oferta do apoio social resultam em situações difíceis para quem está sendo atendido, gerando impacto negativo na vida deste indivíduo(1). Outro estudo realizado com familiares de crianças com necessidades especiais de saúde, também revelou a importância do apoio fornecido pela equipe multiprofissional na atenção à saúde dessa população frente às implicações que a cronicidade desencadeia na vida destes e de suas famílias, especialmente na reabilitação e diminuição das complicações de saúde ${ }^{(16) .}$

Além de desfavorecer a oferta de apoio, a comunicação fragilizada entre a equipe hospitalar traz ainda mais desgastes físicos e emocionais para o cuidador da criança durante o período de hospitalização:

Essa comunicação tem que melhorar e o profissional não deve se sentir tolhido nem invadido quando alguém pergunta, quando alguém questiona. [...] Muitas vezes eu vou fazer a entrevista e eu postergo, porque naquele dia o paciente já passou por vários profissionais (E11).

A comunicação efetiva entre os membros da equipe favorece um olhar ampliado da assistência e possibilita a identificação de demandas a serem trabalhadas por intermédio do apoio. O bem-estar da família no hospital minimiza o desgaste desta e também da equipe, fortalecendo a relação entre família/profissional e a qualidade do cuidado.

Portanto, a equipe precisa elaborar estratégias para estabelecer diálogos mais efetivos entre seus membros e com a família, pois a doença crônica exige olhar diferenciado, que promova o cuidado contínuo e que dê conta de identificar e resolver as alterações provocadas pela doença:

O paciente crônico é um paciente trabalhoso, que precisa ser visto não apenas como uma repetição de condutas, mas que cada dia o olhar fosse colocado não para a doença, mas para o doente (E6).

Em geral, a doença crônica exige variedade de procedimentos e consultas. Isso não pode se tornar um evento em que o cuidado e o apoio fornecido sejam ofertados 
por meio de ações sobrepostas pelos diferentes membros da equipe, mas, todos devem trabalhar na perspectiva da integralidade ${ }^{(11)}$. Portanto, cabe à equipe multiprofissional planejar e desenvolver ações singulares e complementares para que o apoio ofertado vá ao encontro das necessidades da família.

Ademais, a continuidade e a qualidade do fornecimento de apoio social pelos profissionais ao familiar cuidador enfraquecem-se quando a equipe não tem o suporte da instituição para prestar assistência integral. Esse empecilho caracteriza o processo de trabalho em saúde por ações mecanizadas, meramente técnicas, impossibilitando ações humanizadas e o provimento de apoio social à família e à criança/adolescente durante sua permanência no hospital. Quando o profissional não compreende seu papel nesse processo, o cuidado solidário é fragilizado e a família não recebe $\mathrm{o}$ apoio que necessita.

A falta de apoio institucional, muitas vezes, obstaculiza o trabalho dos profissionais, fragilizando a qualidade do serviço e enfraquecendo o reconhecimento da equipe como fonte de apoio pelas famílias durante a hospitalização. Não obstante, a falta de suporte da instituição à família e ao profissional dificulta a continuidade e a qualidade do apoio, implicando em uma assistência frágil e um apoio apenas momentâneo. Nessa perspectiva, identificou-se que a falta de privacidade nas enfermarias, por vezes, causa constrangimentos e limita as ações dos profissionais.

Enfrenta dificuldades com a própria questão do espaço. Às vezes você está atendendo[...] e é difícil porque o espaço é muito pequeno. Às vezes a pessoa [...] se inibe ao falar determinadas coisas porque o companheiro do leito vizinho pode ouvir[...] (E8).

A infraestrutura hospitalar deve garantir conforto, privacidade e segurança para a recuperação da saúde ${ }^{(17)}$ da criança/adolescente. Muitas vezes, a disposição dos mobiliários nas enfermarias, ao invés de facilitar o trabalho dos profissionais, inviabiliza ações de cuidado integral, especialmente àquelas que envolvem a subjetividade da família e da criança/adolescente. Estudo ${ }^{(18)}$ realizado na Inglaterra enfatiza a necessidade de respeitar a confidencialidade e a privacidade da criança/adolescente e família, pois as relações e interações entre crianças, pais e equipe pode ser afetada significativamente pelo modo de organização dos espaços no hospital.

Portanto, é preciso buscar caminhos para superar os obstáculos à formação de vínculos que garantam oferta de apoio social satisfatório às famílias durante a hospitalização de crianças e adolescentes com doença crônica.

\section{Caminhos para superar os obstáculos na formação de vínculos para oferta de apoio social satisfatório}

Dentre os caminhos apontados para superar os obstáculos para a formação de vínculos para oferta de apoio social satisfatório às famílias, os participantes desta pesquisa enfatizaram o seguimento após a alta hospitalar da criança/adolescente. Para isso, destacam a criação de uma equipe ambulatorial especializada para o atendimento e seguimento dessa população, além do incentivo e sensibilização da família sobre a importância na continuidade e realização do tratamento.

[...] minha sugestão, era que tivesse uma equipe montada para paciente crônico ou alguém que fosse a busca de saber como é que esses pacientes estão, se estão correndo bem, se estão tratando, se estão trocando curativo, se estão fazendo fisioterapia [...] (E9).

[...] quando elas saem de alta a gente orientar [...] a importância do retorno para o ambulatório, a importância de não abandonar o tratamento, a importância de marcar as consultas [...] (E13).

O seguimento da criança/adolescente com doença crônica é fundamental no manejo da doença. Para tanto, é preciso garantir uma rede social de apoio às famílias que esteja articulada para reconhecer e atender às demandas de saúde e ofertar apoio satisfatório a essa população. Nessa perspectiva, os vínculos estabelecidos com as famílias são fundamentais, pois estudo ${ }^{(15)}$ adverte que apoiar, educar, aconselhar, fornecer suporte emocional e garantir a continuidade de cuidados às crianças/adolescentes e suas famílias, é a base do cuidado integral na assistência à doença crônica infantojuvenil. Isso implica em colocar a família na perspectiva do cuidado para que a mesma se sinta cuidada e, assim, possa desenvolver habilidades para o enfrentamento da doença crônica contando com uma rede social de apoio fortalecida.

No atendimento intra-hospitalar a equipe de saúde apontou ações que poderiam aproximar família-equipe, fortalecendo vínculo para maior oferta de apoio.

Poderíamos estar sempre orientando, fazendo reuniões, [...] sempre estar conversando com a pessoa, dando apoio de conforto aos pacientes, principalmente àqueles que estão acompanhando, porque eles ali estão se dedicando aos filhos é um dia cansativo, noite e dia sempre ali junto da criança (E7). 
A questão de panfletos, folders, de manuais, de livrinhos com orientação sobre a doença, sobre os cuidados, de tentar mostrar. Tivesse até, assim, um protocolo de como ela seguir quando precisasse de algum encaminhamento, alguma referência, [...] a questão da referência, da contrarreferência, de encaminhar para o serviço especializado, de dizer a importância de essa mãe estar nesse serviço (E12).

Falta o apoio espiritual para essas pessoas que estão fragilizadas, para que elas tenham um conforto, tenha um amparo, um acolhimento maior também do lado espiritual (E6).

Para que se sinta capaz e possa assumir esse cuidado, a família precisa ser informada sobre a doença e o tratamento, preparando-se para o cuidado que será prestado no domicílio. A equipe multiprofissional deve fazer parte da rede social de apoio dessas famílias e, portanto, oferecer o apoio que necessitam para a melhoria da sua qualidade de vida, fortalecendo o bem-estar dessas pessoas. Para isso, precisam estabelecer vínculo para ter a confiança da família na realização do cuidado. Nesse sentido, estudo ${ }^{(19)}$ evidenciou que o apoio fornecido pelos profissionais é recíproco e contribui positivamente para a saúde e bem-estar da família.

Em contrapartida, a ausência dessas ações no espaço hospitalar dificulta o enfrentamento dos cuidadores das implicações trazidas pela condição crônica, uma vez que interfere no funcionamento familiar. Assim, independente da fase da doença da criança/adolescente, a família tem necessidades singulares de cuidado que não podem ficar invisíveis aos profissionais. Somente um vínculo forte da equipe com a família poderá proporcionar caminhos para oferecer o apoio necessário. Nessa direção, estudo(20) evidenciou a relevância de ações centradas na família, bem como a realização de intervenções parentais para auxiliar a família a desenvolver competências e habilidades no cuidado, o que contribui para a redução do estresse parental e melhora do bem-estar.

Além disso, a carência do apoio de reforço, no qual os profissionais reconhecem as habilidades da família no cuidado e no enfrentamento da doença, e do apoio espiritual, para ajudar as famílias a lidar com o desconhecido e sentirem-se apoiadas em suas carências, foram mencionadas pelos participantes deste estudo como lacunas no cuidado. Portanto, evidenciam-se fragilidades que poderiam ser supridas pela equipe ao trabalhar mais próxima do familiar cuidador.

A equipe reconhece as fragilidades no apoio fornecido, porém, vislumbra caminhos para superá-las que precisam ser discutidos pelos próprios profissionais e pelos gestores, para serem implementados no cotidiano do trabalho. Dessa maneira, a equipe estará fortalecendo vínculos com as famílias de crianças/adolescentes com doença crônica durante a hospitalização e o familiar cuidador sentir-se-á apoiado para enfrentar as implicações advindas da cronicidade e revigorado para a luta cotidiana no cuidado a seu filho.

\section{口 CONSIDERAÇÕES FINAIS}

A pesquisa revelou que a equipe multiprofissional emprega estratégias, como a formação de vínculo e acoIhimento para a oferta do apoio social às crianças/adolescentes com doenças crônicas e suas famílias. Porém, o fornecimento de apoio satisfatório às demandas da família ainda se encontra fragilizado tendo em vista problemas decorrentes dos modos como os vínculos são estabelecidos, da falta de infraestrutura hospitalar e do modo de organização do processo de trabalho da equipe multiprofissional.

Em contrapartida, o diálogo e a escuta que promovem o vínculo entre a equipe e as famílias, foram evidenciados como fontes de apoio. Contudo, na prática assistencial ainda existem obstáculos importantes para a formação de vínculo como a organização do processo de trabalho da equipe multiprofissional; fragilidades na interação e comunicação entre os membros da equipe; sobrecarga de trabalho; burocratização dos serviços de saúde; falta de apoio institucional, a serem superados para que a equipe produza um cuidado que responda às necessidades dessas famílias.

Para a prática assistencial este estudo evidencia que a oferta de apoio social e produção de cuidado humanizado e integral às famílias das crianças/adolescentes com doença crônica durante a hospitalização dependerá do vínculo estabelecido entre os profissionais e essa população, principalmente a Enfermagem que permanece 24 horas por dia no hospital. Acredita-se que cuidar da criança/adolescente com doença crônica e sua família implica colocá-los no centro da ação em torno da qual tudo adquire sentido, pois o cuidado humanizado e integral é uma obrigação dos profissionais de saúde, é um dever fundamental e não uma mera opção. Os serviços de saúde precisam focar sua gestão nas políticas e práticas assistenciais que fortaleçam a assistência em saúde humanizada, de qualidade e eficaz. Nessa perspectiva, são necessárias pesquisa que evidenciem as limitações e potencialidades no processo de trabalho em pediatria hospitalar para que a equipe seja fonte de apoio social.

As limitações do estudo estão relacionadas ao fato de este ter sido desenvolvido com um número reduzido de participantes de cada categoria da equipe multiprofissional e em um único hospital que atende crianças e adolescentes com doenças crônicas. 


\section{REFERÊNCIAS}

1. Golics CJ, Basra MKA, Salek MS, Finlay AY. The impact of patients chronic disease on family quality of life: an experience from 26 specialties. Int J Gen Med. 2013 Sep;6:787-98.

2. Instituto Brasileiro de Geografia e Estatística (IBGE). Pesquisa Nacional por Amostra de Domicílios: um panorama da saúde no Brasil, acesso e utilização dos serviços, condições de saúde e fatores de risco e proteção à saúde 2008. Rio de Janeiro: Fiocruz/MS/IBGE; 2010.

3. Geense WW, Van Gaal BGI, Knoll JL, Cornelissen EAM, Van Achterberg T. The support needs of parents having a child with a chronic kidney disease: a focus group study. Child Care Health Dev. 2017 Nov;43(6):831-8. doi: https://doi. org/10.1111/cch.12476.

4. Villas-Boas S, Oliveira AL, Ramos N, Montero I. Apoio social e diversidade geracional: o potencial da LSNS-6. Sips - Pedagogía Social: Rev Interuniversitaria. 2018;31:183-96. doi: https://doi.org/10.7179/PSRI_2018.31.14.

5. Gesteira ECR, Bousso RS, Rodarte AC. Uma reflexão sobre o manejo familiar da criança com doença falciforme. R Enferm Cent 0 Min. 2016 set/ dez;6(3):2454-62.

6. Rodrigues PF, Amador DD, Silva KL, Reichert APS, Collet N. Interaction between the nursing staff and family from the family's perspective. Esc Anna Nery. 2013;17(4):281-7.

7. Reichert APS, Albuquerque TM, Collet N, Minayo MCS. Bond between nurses and mothers of children younger than two years: perception of nurses. Ciênc Saúde Coletiva. 2016;21(8):2375-82.

8. Machado AN, Sousa MLXF, Silva MEA, Coutinho SED, Reichert APS, Collet N. Difficulties in effecting hospital reception at admission of children with chronic disease. Rev Enferm UERJ. 2015;23(4):556-61.

9. Minayo MCS. 0 desafio do conhecimento: pesquisa qualitativa em saúde. 14a ed. São Paulo: Hucitec; 2014.
10. Fontanella BJB, Luchesi BM, Saidel MGB, Ricas J, Turato ER, Melo DG. [Sampling in qualitative research: a proposal for procedures to detect theoretical saturation]. Cad Saúde Pública. 2011;27(2):389-94. Portuguese.

11. Pereira MM, Rodrigues PF, Santos NCCB, Vaz EMC, Collet N, Reichert APS. Educação em saúde para famílias de crianças/adolescentes com doença crônica. Rev Enferm UERJ. 2017; 25:e4343. doi: https://doi.org/10.12957/reuerj.2017.4343.

12. Azevêdo AVS, Lançoni Junior AC, Crepaldi MA. Nursing team, familyand hospitalized child interaction: an integrative review. Ciênc Saúde Coletiva. 2017;22(11):3653-66.

13. Neves ET, Buboltz FL, Silveira A, Kegler JJ, Silva, JH, Santos RP, Zamberlan KC. [Network of support for families of children in pediatric emergency department]. Rev Pesq Qualit. 2017;5(7):53-65. Portuguese.

14. Macedo IF, Souza TV, Oliveira ICS, Cibreiros SA, Morais RCM, Vieira RFC. Nursing team's conceptions about the families of hospitalized children. Rev Bras Enferm. 2017;70(5):904-11.

15. Polita T, Tacla MTGM. Network and social support to families of children with cerebral palsy. Esc Anna Nery. 2014;18(1):75-81.

16. Barbosa TA, Reis KMN, Lomba GO, Alves GV, Braga PP. [Network and social support of families of children with chronic conditions]. Rev Rene. 2016 janfev; $7(1): 60-6$. Portuguese.

17. Neves FG, Moraes JRMM, Morais RCM, Souza TV, Ciuffo LL, Oliveira ICS. Nursing work in pediatric emergency from the perspective of the companion. Esc Anna Nery. 2016;20(3):e20160063. doi: https://doi.org/10.5935/1414-8145.20160063.

18. Curtis P, Northcott A. The impact of single and shared rooms on family-centred care in children's hospitals. J Clin Nurs. 2017;26(11-12):1584-96.

19. Pennafort VPS, Queiroz MVO, Nascimento LC, Guedes MVC. Network and social support in family care of children with diabetes. Rev Bras Enferm. 2016;69(5):856-63. doi: https://doi.org/10.1590/0034-7167-2015-0085.

20. Crnic KA, Neece CL, McIntyre LL, Blacher J, Baker BJ. Intellectual disability and developmental risk: promoting intervention to improve child and family wellbeing. Child Dev. 2017;88(2):436-45.

\section{Autor correspondente:}

Amanda Narciso Machado

E-mail: amandanmachado@hotmail.com
Recebido: 16.01.2018

Aprovado: 16.04.2018 